\title{
Crítica literaria, Romanticismo y esfera pública en el Semanario Pintoresco Españo/ 1
}

\author{
Literary Criticism, Romanticism and the Public \\ Sphere in the Semanario Pintoresco Español
}

\author{
Luis Alejandro Nitrihual Valdebenito \\ Juan Manuel Fierro Bustos \\ Orietta Geeregat Vera ${ }^{2}$
}

\section{Resumen}

El presente trabajo expone las articulaciones teóricas que sustentan un estudio acerca de la crítica literaria desarrollada en medios de prensa. Se plantea una discusión teórica a partir de autores como Terry Eagleton, Jürgen Habermas, Albert Chillón y otros que han elaborado un planteamiento sobre el tema. Asimismo, se busca situar la discusión en el contexto de la crítica aparecida en el Semanario Pintoresco Español.

Palabras claves

Crítica literaria, Ilustración, medios de prensa, esfera pública.

\section{Abstract}

This paper presents theoretical joints underlying a study on the literary criticism developed in press. There is a theoretical discussion from authors such as Terry Eagleton, Jürgen Habermas, Albert Chillón and others that have developed an approach on the subject. Also seeks to place the discussion in the context of which appeared in the Semanario Pintoresco Español, critique.

Key words

Literary criticism, Illustration, press, public sphere.

Artículo recibido el 6 de Julio de 2014 y aprobado el 20 de agosto de 2014

1 Artículo producto del proyecto de investigación fondecyt 1141289, financiado por el Consejo Nacional de Ciencia y Tecnología. Los autores de este artículo son parte del Centro de Investigación Comunicación, Discurso y Poder. Núcleo Científico de Ciencias Sociales. Universidad de La Frontera.

2 Universidad de La Frontera, Facultad de Educación, Ciencias Sociales y Humanidades, Departamento de Lenguas Literatura y Comunicación, Centro de Investigación Comunicación, Discurso y Poder. Chile. Correo electrónico: luis.nitrihual@ufrontera.cl 


\section{Propósitos de la investigación}

Siempre es una empresa interesante y riesgosa escribir un artículo de un objeto tan específico como el que proponemos en esta investigación: el Semanario Pintoresco Español. ${ }^{3}$

Es riesgosa fundamentalmente por cuanto para describir con propiedad un periódico, un diario, un semanario o cualquier medio de comunicación es necesario adentrarse en la estructura política y económica que la sustenta en un determinado momento histórico.

Ahora bien, aun con la limitación que nos impone el contexto, podemos reconocer ciertos recorridos, lenguajes y saberes comunes que hacen de los textos "tejidos" que se conectan y que permiten observar algunas estructuras de sentimiento (Williams, 2003) comunes a distintas realidades socio-históricas.

Ahora bien, hay que considerar las particularidades que asume la estética en relación con la totalidad social y los contextos históricos específicos. Por ejemplo, son evidentes las diferencias de manifestaciones estético-políticas como el Romanticismo en Europa y América. Incluso, las diferencias entre el Romanticismo francés y el español hacen que cada uno tenga sus propias particularidades. En el caso latinoamericano, por su parte, toda manifestación cultural del siglo XIX se encuentra cruzada (determinada) por la coyuntura de la independencia política y la posterior búsqueda de la independencia cultural (alejamiento del ancien régime y construcción de un espacio cultural autónomo).

En consecuencia, nos proponemos, en primer término, describir las características de la crítica cultural, su relevancia en el marco de formaciones discursivas propias de los albores de la modernidad y de la cultura de masas. En un segundo momento, buscaremos sustentar la posibilidad de emprender investigaciones que relacionen la literatura y el periodismo. En este último aspecto, pensamos que ambos géneros, hoy separados de manera tajante, en medios de prensa como el Semanario se encuentran

3 Desde este punto lo denominaremos sencillamente Semanario. imbricados y hacen que su lectura sea de una actualidad, vigencia y riqueza fascinante.

En un tercer momento, veremos algunas especificidades del Semanario, fundamentalmente observaremos la manera en que en sus fascículos se muestra, de modo ejemplar, la construcción de un nuevo tipo de espacio donde habitar como proyecto país, de una emancipación de la cultura y de la literatura como un ámbito desde el cual pensar la construcción de los nacientes estados.

Por último, buscaremos establecer algunas correlaciones entre el desarrollo del Romanticismo en Chile y el español, fundamentalmente a través del trabajo crítico del intelectual chileno José Victorino Lastarria. Asimismo, relevaremos la figura de Lastarria no solo como literato sino también como fundador de la prensa político-literaria, cuestión que nos interesa pues traza el rumbo de las investigaciones que venimos desarrollando desde hace unos años. Junto a su labor literaria, el intelectual criollo dirigió, editó y tuvo participación en decenas de periódicos que pensaron la cultura y la literatura, no solo como un modo de representar la realidad sino también como una herramienta de desarrollo social. Pensamos que esto sella, en el siglo XIX, la estrecha relación entre el surgimiento de los géneros literarios y el posterior desarrollo de los géneros que la periodística se ha esforzado en delimitar, pero que, en el caso de la prensa de los siglos XVIII, XIX y primeros del XX, es inexistente y más bien estamos en presencia de textos intergenéricos.

\section{Crítica, ilustración y cultura de masas}

La crítica es un fenómeno discursivo propio de la Modernidad. Como tipo discursivo posee una larga tradición en la formación de los sistemas mediáticos (Nitrihual, 2006, 2007; Nitrihual, Fierro, Mayorga, Del Valle, 2011). Justamente, apuntando a esta última idea, Hallin y Mancini (1998) en su libro: Sistemas mediáticos comparados definen y describen un "sistema mediático mediterráneo" caracterizado por su vínculo con la literatura y la política. España e Italia, en Europa, compartirían estas características. 
Ahora bien, puede visualizarse el desarrollo de la crítica en dos grandes recorridos: 1. Desde la teoría literaria y, como soporte, desde las revistas especializadas; 2 . Estrechamente vinculado al ejercicio periodístico, teniendo como soporte, los medios de prensa.

Apuntando a la crítica desarrollada en medios de prensa, Jürgen Habermas (2006) destaca la idea de que esta contribuyó a conformar la esfera pública al tratarse del diálogo racional entre un crítico y su "público masivo". La crítica surge, de este modo, como una nueva estrategia de racionalidad en el marco de la Modernidad.

Terry Eagleton (1999) señala, por su parte, que la crítica ha desempeñado las siguientes funciones históricas:

1. Durante el siglo XVIII, la crítica tuvo que ver con política cultural. Las revistas de esta época tienen un carácter cultural, no propiamente literario. El hecho literario le sirve al hombre ilustrado más bien como pretexto para hablar de normas, valores, situaciones sociales como el problema de la mujer, etc. (Eagleton, 1999, p. 22). Este hombre de letras, cuya figura se perfila ya claramente en la segunda mitad del siglo, desempeña una función orgánica de articulación entre el público masivo y los medios de prensa.

2. En el siglo XIX la crítica tuvo una preocupación por la moralidad pública. En este siglo se concreta la figura del "hombre de letras". Este estamento intelectual se encuentra vinculado con los medios de comunicación y con la industria editorial. Podría pensarse, desde la perspectiva de Antonio Gramsci, que este segmento intelectual surgió como parte de la intelectualidad orgánica producida por la burguesía mercantil.

3. La crítica literaria durante el siglo XX se arroja hacia una cuestión específicamente literaria, aunque con la deformación que introduce en la esfera pública la primacía el mercado.

Un desarrollo paradójico tiene la crítica literaria: su existencia surge como parte de la publicidad literaria, de la necesidad de diálogo racional en la etapa de formación de la esfera pública. No obstante, una vez mercantilizada la cultura, la crítica se refugia en la especialización de los campus universitarios, por un lado, y en la reseña de libros en el caso de los medios de prensa, por otro.

Un aspecto interesante de la crítica, asimismo, es que ha sido un espacio de discusión de lo político. En el siglo XVIII, a través de la crítica, se producían enconadas discusiones que revelaban las tensiones y las luchas de las clases sociales. Por esta razón, los intelectuales aspiraban participar en la prensa. Como destaca Fernández (2006, p. 278):

De manera similar a lo que sucedía en Francia, una de las principales aspiraciones de los escritores españoles en la segunda mitad del diecinueve era ocupar un puesto en la organización política de la sociedad y el camino para lograrlo solía ser entrar a formar parte de la redacción de un periódico de prestigio social y, gracias a los contactos que allí se establecían y al apoyo de algún político, obtener un cargo oficial para acabar convirtiéndose en hombre público o, en el caso de los poetas, ver su obra publicada.

Ahora bien, durante el siglo XIX y XX, la crítica tiene una separación tajante con su público. Si la sociedad moderna se entiende a través de discursos, modas y personalidades reconocidas (como son los críticos literarios), tanto el reconocimiento como el desprecio constituyen una norma (Sloterdijk, 2002, p. 32):

Si el mundo moderno, tal como han expuesto de manera razonable ciertos intérpretes de Hegel, se define por ser un lugar de enfrentamiento de luchas generalizadas por el reconocimiento, este tiene que conducir inevitablemente a una forma de sociedad en la que el desprecio alcanza cotas endémicas.

Entonces, es posible proponer que la paulatina desaparición de la crítica en la actualidad — esa idea ilustrada de educar a las masas, propia del pensamiento de figuras como José Victorino Lastarria o Jotabeche en Chile o del pensamiento de Larra o Mesoneros Romano en España- se hunde cuando el mercado y el culto a la individualidad desarrollan su fuerza sísmica y transformadora de una práctica discursiva que constituyó una red fundamental para el modelamiento del espacio público. 


\section{Romanticismo, Modernidad e llustración en el Semanario Pintoresco Español}

La primera mitad del siglo XIX es particularmente importante en la conformación de un espacio público raciocinante, en el surgimiento de ciertas estéticas y en la formación del sistema mediático. Todo esto, evidentemente, hay que enmarcarlo en la evolución del capitalismo y su irrupción en la gestación de los modernos medios de comunicación, cuya financiación por medio de la publicidad genera grandes cambios dentro de ellos, como señala De las Heras Pedrosa (2000), la masificación de la prensa debido a la baja de sus precios (dentro de otras variables, pues también hay que considerar el paulatino proceso de alfabetización que consolida una masa lectora).

Una lectura inicial del Semanario resulta sumamente interesante por su heterogeneidad. El valor de esta publicación radica, entre otros aspectos, en la riqueza de un lenguaje aún no dividido en géneros narrativos. Con razón, Rubio (1995) ha señalado que el Romanticismo produce en España una revitalización de los géneros narrativos gracias a la utilización de los periódicos como medios de difusión. Las características de estos medios, que como ya hemos visto en los párrafos anteriores se ubican en la etapa de formación de la Modernidad y del nacimiento de la cultura de masas, serían la introducción de secciones muy diversas que van desde novelas, cuentos, leyendas, artículos de costumbres y, aunque no en un primer momento, crítica literaria. La mejor descripción de la variedad de temáticas y géneros que aparecen en el Semanario la realiza el propio Ramón Mesonero Romano en el prospecto inicial:

No seguiremos orden metódico en la elección de materias; buscaremos en el estudio de la naturaleza, de las bellas artes, de la literatura, de la industria, de la historia, de la biografía y de las costumbres antiguas y modernas, todos los hechos, todos los adelantos capaces de interesar curiosidad pública; procuraremos dar unos consejos útiles y aplicables a las distintas profesiones sociales, intentaremos distraer a otras de sus fatigas por medio de narraciones interesantes. (Semanario, Prospecto, p. 3)
Descomponiendo este párrafo se encuentran algunas claves para comprender el inicio de la prensa propiamente moderna. Señalaremos cuatro aspectos: 1) masificación de la lectura de los periódicos como elemento de desarrollo profesional y personal; 2) presencia de textos que buscan sentar las bases de una moral y unas costumbres nuevas; 3 ) preocupación por los hechos y acontecimientos; y 4) una faceta de entretenimiento no menos relevante y que se revelará de ahí en más, hasta hoy, en los medios modernos de comunicación. Sin más: educación, información y entretenimiento. Claves fundantes de los medios de comunicación modernos.

Nos gustaría resaltar el segundo punto, que es al que menos atención se le presta: el Semanario describe muy bien sus quehaceres en términos de lo moral, lo público y lo político: "Procuraremos no desatender la moral pública y privada, cuyo ejercicio práctico une a los hombres en sociedad [...] los deberes religiosos y civiles, la tolerancia, el amor al trabajo".

No olvidemos lo que habíamos señalado en torno a que la crítica durante el siglo XIX tuvo una preocupación por la moralidad pública. Basta recordar cómo en el primer número del Semanario hay un artículo titulado "Moral privada", cuyo párrafo inicial señala: "La moral es una planta cuya raíz está en los cielos, y cuyas flores y frutos perfuman y embellecen la tierra" (Semanario, 1, p. 12).

Por otro lado, una característica central de los periódicos de la época es que se encontraban marcados por la Ilustración. Se trata de medios que tenían finalidades educativas de carácter popular y masivo. Como se reitera en el prospecto del Semanario: "En nuestra España acaso no se ha escrito más que para un número muy reducido de personas. Muchos discursos altisonantes, muchos terribles infolios; pero el pueblo ni puede costear infolios, ni comprende erizadas disertaciones". Esto ha sido resaltado por Isabel Tajahuerce Ángel (1996), quien destaca el carácter masivo de esta publicación: "El Semanario Pintoresco está destinado a un público más numeroso y no excesivamente culto, por eso es necesario utilizar un estilo que esos lectores puedan comprender, además de vender el periódico a un precio asequible". 
En su artículo titulado "Aproximación a un estudio de las reseñas literarias en diversos suplementos culturales españoles", los autores Ana García y Mikhael Muro (2011) han descrito un breve panorama del surgimiento de la crítica en los suplementos españoles. Para estos autores, un periodo clave en su desarrollo es 1833, tras la muerte de Fernando VII.

Ahora bien, aunque estos autores no desarrollaron una mirada teóricamente profunda sobre la formación de la crítica literaria, se encuentra clarificado que esta surgió como reacción a los Estados absolutos. De este modo, resulta clave la relevancia que toma la crítica, luego de la muerte de Fernando VII, en la configuración de la espera pública.

Por otro lado, la profesionalización de la actividad intelectual es clave. Los periódicos aparecen como posibilidades de sustento económico. Nuevamente, nos encontramos con los albores de la Modernidad, siempre inseparable del desarrollo del capitalismo. Por esta razón, aunque no lo exploraremos en este trabajo, debemos comprender el fenómeno del surgimiento de estéticas como el Romanticismo, o las propias vanguardias, como parte de una evolución más general de cierto modelo de sociedad.

Es necesario, entonces, consignar que en la conformación del sistema mediático, tanto en España como en Chile, los medios de comunicación se desarrollaron a partir de las instituciones literarias y políticas. Aunque el mercado aparece con fuerza durante el siglo XX y lo que va del XXI, durante los siglos anteriores ha sido un sistema con un alto paralelismo político (vinculado a partidos políticos) y también por haber nacido bajo los fines ilustrados de la institución literaria (Hallin y Mancini, 1998, p. 91): "Como indica la historia, los medios de comunicación en los países mediterráneos están considerablemente politizados, y el nivel de paralelismo político es relativamente alto. El estilo periodístico tiende a poner un importante énfasis en el comentario".

En este panorama de formación, la relación arte/ mercado se consolida. Por ejemplo, surge la aspiración ilustrada de una literatura que se encuentre al alcance de todos. Esto queda claramente establecido en el primer número del Semanario, cuando Ramón Mesoneros Romano señala:
Muchas invenciones, muchos adelantos se han hecho en el siglo actual en otros países; pero ni las máquinas de vapor, ni lo globos, ni el gas, ni los caminos de hierro, ni tantas aplicaciones útiles para la industria, ha producido al pueblo mayor beneficio que las publicaciones baratas.

Igual fenómeno se dará con José Victorino Lastarria en Chile, quien, como veremos luego, en su texto fundacional de la Sociedad Literaria de 1842, arranca de las mismas consideraciones que Mesoneros. Asimismo, Ana García y Mikhael Muro (2011) destacan que otra de las figuras claves en la fecunda relación entre literatura y periodismo en España es Mariano José de Larra. No es extraño, siguiendo con los paralelismos, que Lastarria en Chile señale, entre los pocos intelectuales que reconoce como modelo en España, a Larra como un referente.

\section{Chile y España, siglo XIX. A modo de anticipo y cierre}

José Victorino Lastarria es un personaje capital en la historia cultural y política chilena. Nacido en 1817 en la ciudad de Rancagua, estudió en el Instituto Nacional y recibió su título de abogado en la Universidad de San Felipe. Es considerado el verdadero impulsor de la literatura chilena y, agregaríamos nosotros, se inicia con él una fructífera relación entre literatura y periodismo.

El pensamiento de Lastarria surge en un contexto de instalación del pensamiento ilustrado que busca construir un espacio cultural (literario y mediático) autónomo desde el cual pensar la República e instalar preocupaciones fundamentales acerca de la independencia cultural del país y fijar los fundamentos de la identidad nacional. Ahora bien, esta construcción es realizada desde un distanciamiento crítico con el periodo colonial y sus enclaves. Empero, para que esto fuera posible, Lastarria se encuentra con una floreciente cultura letrada. Su quehacer intelectual se transforma así y se proyecta como resultado, hasta el día de hoy, de un quehacer críticamente fundacional y fronterizo; como también lo fue en un contexto socio-político y cultural distinto, el propósito del Semanario Pintoresco en España. 
Los rasgos característicos de la obra periodística de Lastarria, que lo separan de figuras tan reconocidas como Andrés Bello, son: a) la relevancia que otorga los medios de prensa en la construcción de la esfera pública y su estratégica función en los procesos de consolidación del Estado chileno; y b) la radical separación del ancien régime que muestra en su pensamiento. La difusión de la literatura y la prensa es para Lastarria un instrumento de educación para el pueblo. Su ideario estético-político constituye un pensamiento libertario de absoluta vigencia en Chile.

Tanto Mesoneros Romano como Lastarria pretenden no solo escribir, sino levantar propuestas de creación de medios de prensa para romper la inercia cultural, en sus respectivos contextos, con propósitos divulgadores y democratizadores, ampliando de esta manera la cobertura de destinatarios sin distinciones de clase. Ambos confiaban en la dimensión performativa del discurso público, operacionalizado en medios literarios tradicionales y periodísticos emergentes. La siguiente cita de Lastarria muestra la vigencia de estos fundamentos y posibilita que tal noción pudiera estar perfectamente contenida en cualquier periódico ubicado temporalmente en la primera mitad del siglo XIX:

Se dice que la literatura es la expresión de la sociedad, porque en efecto es el resorte que revela de una manera más explícita las necesidades morales e intelectuales de los pueblos, es el cuadro en que están consignadas las ideas y pasiones. (Lastarria, 1842 en Promis, 1995, p. 82)

Lastarria percibe con gran claridad los rasgos constituyentes de los sectores dominantes en Chile y sus arraigos, las contradicciones, los vicios y virtudes que determinan el destino de la nación, cuestión que él necesita denunciar públicamente y cuya utopía es aclarar las contradicciones de una sociedad que necesita redefinir sus propósitos. Hoy podemos apreciar que su visión crítica y visionaria permanece vigente en muchos de sus planteamientos, ante invariantes sociales y culturales presentes en Chile como condiciones aún no superadas. Al igual que en la época de Lastarria, los medios de comunica- ción sustentan su discurso en la develación crítica de las costumbres y de los vicios de la sociedad así como en la necesidad de crear conciencia amplia en los ciudadanos para posibilitar la transformación personal y social.

En suma, como se puede observar, la crítica representa aquí, al igual que en el Semanario, un instrumento con el que se construye un espacio cultural que se pretende propio. Los medios de prensa, asimismo, son parte de una comunidad discursiva que articulará la construcción de un espacio público fragmentado por las luchas sociales.

\section{Referencias}

Chillón, A. (1999). Literatura y periodismo: Una tradición de relaciones promiscuas. Barcelona: Universitat Autónoma de Barcelona.

Eagleton, T. (1999). La función de la crítica. Barcelona: Paidós.

Fernández, S. (2006). Periodismo y Literatura: una contribución a la delimitación de la frontera. Revista Estudios del Mensaje Periodístico, (12), 275-284.

García, A., y Muro, M. (2011). Aproximación a un estudio de las reseñas literarias en diversos suplementos culturales españoles. Philológica Urcitana, 4, 79-87.

Guerrero, E. (1995). Reflexiones de un crítico. En M. Nieves, M. Rodríguez, G. Triviños (eds.), La crítica literaria chilena (pp. 113-117). Concepción: Anibal Pinto.

Habermas, J. (2006). Historia y crítica de la opinión pública. La transformación estructural de la vida pública. Barcelona: Gustavo Gili.

Hallin, D., y Mancini, P. (2007). Sistemas mediáticos comparados. Madrid: Hacer Editorial.

Heras de las, C. (2000). El papel de publicidad en la empresa periodística. Málaga: Universidad de Málaga.

Nitrihual, L. (2006). La crítica literaria de Alone y su relación con la problemática de género. Una mirada a la obra de Gabriela Mistral y Marta Brunet. Revista del Grupo de Investigación Escritoras y Escrituras, (4), en línea: http://www.escritorasyescrituras.com/ revista.php/4/31

Nitrihual, L. (2007). La crítica literaria de la Revista de Libros del diario chileno El Mercurio, entre los años 2002-2004. Revista Palabra Clave, 10(2), 135-145. 
Nitrihual, L., Fierro, J. Manuel, Mayorga J., Del Valle, C. (2011). Crítica y literatura en José Victorino Lastarria. Ancien Regime e Ilustración. Revista Historia y Comunicación Social, 16, 97-110

Promis, J. (1995). Testimonios y documentos de la literatura chilena. Santiago de Chile: Editorial Andrés Bello.

Rivera, J. (1995). El periodismo cultural. Buenos Aires: Paidós.

Sloterdijk, P. (2002). El desprecio de las masas. España: Pretexto.

Tajahuerce, I. (1996). El arte en las revistas ilustradas madrileñas. 1835-1840 (Tesis para optar al grado de Doctor). Universidad Complutense de Madrid.

Williams, R. (2003). La larga revolución. Buenos Aires: Nueva Visión. 